\title{
VISUAL QUERYING IN GEOGRAPHIC INFORMATION SYSTEMS
}

\author{
Valéria Gonçalves Soares \\ Federal University of Pernambuco \\ Agriculture and Livestock Research Company of Rio Grande do Norte State \\ vgs@cin.ufpe.br,valeria@dmrh.emparn.br
}

Ana Carolina Salgado

Federal University of Pernambuco

acs@cin.ufpe.br

\begin{abstract}
This article presents a framework that defines a visual query language for Geographical Information Systems (GIS). The main objective of the proposed framework is to improve the friendliness of a GIS interface, which is achieved by using pictorial information to build query clauses and by reducing the differences among GIS internal structures. The queries use visual elements and visual spatial operators, which are represented according to a Geographic Visual Query Language, the GeoVisualQL.
\end{abstract}

Keywords: Geographic Information Systems (GIS), Visual Query Language.

\section{INTRODUCTION}

A Geographic Information System - GIS is "a powerful set of tools for collecting, storing, retrieving at will, transforming and displaying spatial data from a particular set of purposes" (Burrough and McDonnell. 1998). Geographical data represent phenomena from the real world in terms of their position in coordinate systems, their attributes and their spatial interrelations with each other.

User interfaces for GIS require considerable research and technology development efforts for usability enhancement (Oliveira, 1997) (Wessel and Haarslev, 1999). An important aspect of usability in this context is the need 
for a visual query language to enable users to think graphically, while building queries. Most current GIS are able to generate graphical representations of query results, but only a few systems support graphical queries (Danko, 2000). It is difficult for the typical GIS users to formulate queries using complex spatial operators and related entities in a textual form. This difficulty can be reduced by the use of Visual Query Languages (VQL) (Calcinelli and Mainguenaud, 1994) and it is the motivation behind using visual queries in GIS.

Generally GIS interfaces have several differences from each other and they may offer too complex mechanisms to define queries. The proposed framework uses a Geospatial Metadata Standard (FGDC Standards, 1999) and Spatial Query Language (Open GIS Consortium, SQL, 1999) concepts to define a Geographic Data Visual Standard (GeoVisual Standard). The motivation behind using this GeoVisual Standard is designing user interfaces that are rather easier to use by improving data visualization and integration. This new standard is defined based on visual elements and spatial operators and is used to define the Geographic Visual Query Language (GeoVisualQL). The proposed GeoVisualQL is, in turn, syntactically and semantically defined to support the main Spatial Query Language structures.

Users will not need to learn the internal structures and the query language for each GIS being used. Instead, they will use the same visual language, based on metadata standard, to query several GIS. The proposed framework supports not only visual queries by the direct manipulation of visual elements, but also enables users to navigate through the metadata information at the interface, before building the queries.

The remainder of this paper is structured as follows. The next section presents some information about Visual Query Systems. Section 3 presents a discussion on Spatial Metadata Standards. Section 4 discusses on how spatial metadata can be useful in visual querying. Section 5 presents the proposed GeoVisual Query Language. Section 6 presents some related work. Finally, in section 7, the current status of our work is presented.

\section{VISUAL QUERY SYSTEMS}

Visual Query Systems (VQS) are database query systems that use a visual representation to depict the domain of interest and to express related requests (Catarci, et al. 1997). In addition to providing the capability to express queries in a visual form, these systems also provide different functionality to enhance human-computer interaction. The use of visual tools can help users in accessing databases without requiring previous learning on a specific query language. 
The goal of a typical VQS user is to retrieve the desired data by developing two main activities: understanding the target domain and formulating the query. The query formulation can be done by four strategies that identify the VQS type: by schema navigation; by sub-queries, by matching and by range selection (Catarci, et al. 1997).

\subsection{Visual Formalisms}

The term visual language is used to describe several types of languages: languages for manipulating visual information; languages for supporting visual interaction and languages for programming with visual expressions (Golin and Reiss, 1990).

When we talk about visual languages we mean that they are completely different from textual languages in terms of their formalization. For example, consider a weather map: sometimes it is not clear how its syntax or semantics can be specified, or even if it has sharp and well-defined boundaries (Marriot and Meyer, 1998).

There are different approaches for specifying visual languages: the grammatical approach, that is based on grammatical formalisms used for the specification of textual languages; the first-order mathematical approach that uses forms of mathematical logic which often stem from artificial intelligence; and the algebraic approach that consists of composition functions which construct complex pictures from simpler pictorial elements.

After having analyzed different grammar approaches that can be used to specify visual languages (Gooday and Cohn, 1996) (Haarslev, 1995)(Marriot and Meyer, 1998) (Wang and Zeevat 1998) we have finally decided to use the Picture Layout Grammar - PLG (Golin, 1991) (Golin and Reiss, 1990), because it better satisfies our goals.

\section{SPATIAL METADATA STANDARDS}

Metadata describes the content, quality, condition and other relevant data characteristics (Hart and Phillips, 1999). The concept of metadata is familiar to most people who deal with spatial issues. A map legend, for example, is pure metadata (The Value of Metadata, 1999). Nowadays, most digital geospatial files have some associated metadata.

The Federal Geographic Data Committee (FGDC Standards, 1999) adopted a content standard for metadata, the Content Standard for Digital Geospatial Metadata (CSDGM), that is a spatial metadata standard developed to support the National Spatial Data Infrastructure (NSDI) (Tosta, 1998). The NSDI was created in order to coordinate data acquisition and 
provide access to geospatial data in USA. The Content Standard provides a consistent approach and format for the description of data characteristics.

In Europe, some initiatives that trend to gather research efforts shall be mentioned. A lot of different metadata formats and information systems are emerging all over Europe (Balfanz and Göbel, 1999) for example, CEN ENV 12657 and UDK 4.0. But both of them do not have neither levels of compliance or any methodology to extent the original metadata model. These are the main reasons for having used the Content Standard of FGDC in this work.

\section{VISUAL QUERIES USING SPATIAL METADATA STANDARDS}

We present a solution to the problem of querying GIS, especially in terms of their user interface. As mentioned above, Geographical Information Systems often do not provide friendly interfaces (Oliveira and Medeiros, 1996) (Oliveira, 1997). Generally the Query Language is specific and dependent on the Geographic Database internal structure. It is known that query formulation in traditional Spatial Query Languages (Egenhofer, 1992) is difficult because their structures are too complex. Typical users do not have the necessary knowledge of the language to express their real needs in a consistent manner (Oliveira and Medeiros, 1996) (Oliveira, 1997). Textual query clauses having several spatial entities and spatial operators related to each other are usually extremely confusing because spatial information is essentially visual.

The main objective of the proposed framework is to be as general as possible, so that the same interface can be used in several different GIS. For this purpose, users should be allowed to use exactly the same query format in any GIS.

\subsection{Why Use Spatial Metadata?}

The use of spatial metadata is important because we want to present geographic information from various GIS by always using the same format. In addition, the main objective of Spatial Metadata Standards, such as FGDC (FGDC Standards Reference Model, 1999), is to make spatial data clear and sharable.

The idea is to use a Standard for Digital Geospatial Metadata such as CSDGM (FGDC Standards Reference Model, 1999), because it allows users to describe several types of geographic entities by using its formalisms. Then, we aim to define a Geographic Visual Standard, the GeoVisual Standard, for 
several types of geodata by applying some of these spatial metadata definitions and based on the conceptual model of the OpenGIS Consortium (OGC) (OpenGIS Consortium, 1999).

We will represent spatial entities that are manipulated in GIS by visual elements in the GeoVisual Standard. In general, these visual spatial elements are represented by the following geometric entities: point, line and region.

This new visual standard describes many kinds of geographic elements, and, for each of these elements, their spatial operators allowed. The GeoVisual Standard defines how to describe geographic data visually. All the spatial entities that are manipulated in GIS are also represented by visual elements at the interface.

The Geographic Visual Query Language (GeoVisualQL) is defined using visual elements and spatial operators with syntactic rules based on the SQL Specification of the Open GIS Consortium (Open GIS Consortium, SQL, 1999). Spatial operators are also graphically represented in our system to allow users to compose queries in a completely visual form.

The syntax of GeoVisualQL was defined based on the SQL specification of the Open GIS Consortium (Open GIS Consortium, SQL, 1999). This allows all possible SQL structures to be constructed in the visual language as well. Before choosing the Spatial Query Language to be used in this definition we have done a comparative study about some of these languages (Egenhofer, 1994) (Sambura, 1998).

\section{THE GEOVISUAL FRAMEWORK}

The GeoVisual Framework is composed basically of four modules: GIS Components, Metadata Model, Query Manager, and Graphic Interface, as shown in Figure 1. All these modules are described in (Soares and Salgado, 2000) (Soares, 2002). In this paper we will discuss only the Query Manager Module.

The Query Manager Module is composed by the Geographic Visual Query Language - GeoVisualQL, and the Query Translator that converts the visual query to a textual query based on the SQL specification of the Open GIS (Open GIS Consortium, SQL, 1999). We will, now, present the GeoVisualQL. The Query Translator is under specification and will not be presented in this paper. 


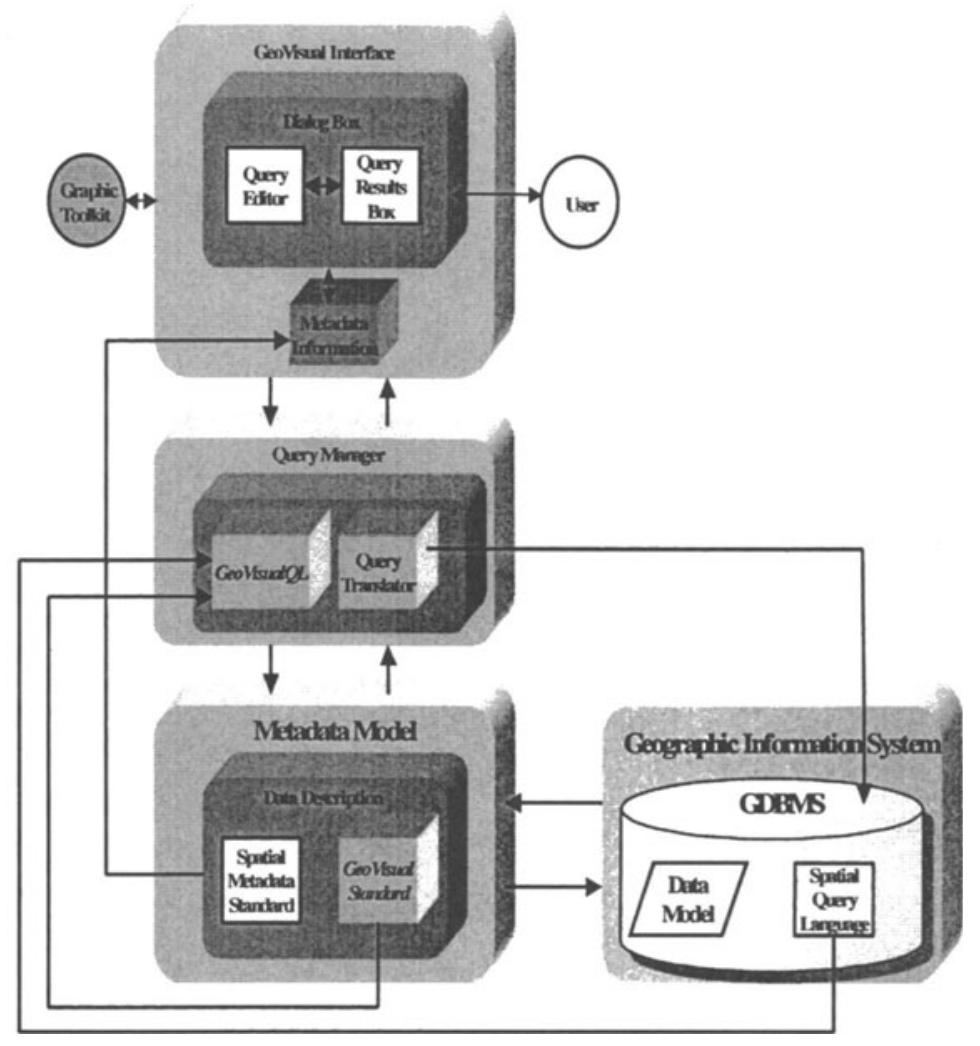

Figure 1 GeoVisual Framework

\subsection{The GeoVisualQL}

As stated before, the Geographic Data Visual Query Language GeoVisualQL, is based on the SQL specification of the Open GIS Consortium (Open GIS Consortium, SQL, 1999). In order to formalize this language, some definitions have had to be specified:

Definition 5.1: The GeoVisualQL is a triple $G Q L=(O, R, \Sigma)$, where $O$ is a set of graphic primitives that represent geographic objects, $R$ is the set of spatial relationships allowed and $\Sigma$ is the set of restrictions.

Pictorial Objects are geographic pictoric entities identified and with attributes. They can participate of spatial relationships that define relationships between objects and sub-objects.

Definition 5.2: A pictorial Object in GQL is a triple gql=(id,o,l) where id $\varepsilon \mathrm{ID}$ is the object identity derived from the set of identifiers $\mathrm{ID}, o \varepsilon \mathrm{O}$ is its type and 1 (eventually null) is the values list of its attributes. 
Definition 5.3: A pictorial relationship in GQL is a 6-tuple $\mathrm{rp}=$ (r,po1,po2,s1,s2,s3), where $\mathrm{r} \varepsilon \mathrm{R}$ is the spatial relationship type, po1 and po2 are both object identifiers or variable names and each sj (eventually null) is a set of object identifiers or variable names.

Based on the above definitions, we will define the components that are necessary to the GeoVisualQL specification.

\subsubsection{Pictorial Objects}

The pictorial objects of the GeoVisualQL and the Geographic Data Visual Standard, GeoVisual Standard are intrinsically related to the chosen spatial data model (Open GIS Consortium, 1999). The primitive graphic elements (i.e. the pictorial objects of GeoVisualQL) are based on the SQL Specification of the Open GIS Consortium Geometry Model (Open GIS Consortium, SQL, SQL, 1999): point, curve, line-string, line, line-ring, polygon, multicurve, multipolygon, multiline-string and multipoint.

\subsubsection{Spatial Relationships}

The Spatial Relationships and Spatial Operators used in GeoVisualQL with their respective restrictions are presented as follows. Consider the term any to represent any pictorial object defined above.

Definition 5.4: The following spatial relationships, and the type returned from each function compose the GeoVisualQL Language:

Equals (any, any) : integer

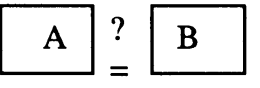

Touches (any, any) : integer

A $\left[\begin{array}{l}B \\ \hline\end{array}\right.$

Disjoint (any, any) : integer

A

B

Within (any, any) : integer

$\mathrm{B}$ 
Overlaps (any, any) : integer

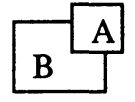

Crosses (any, any) : integer

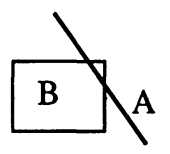

Intersects (any, any) : integer Contains (any, any) : integer

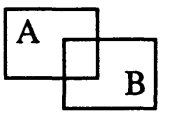

$$
\text { A B }
$$

Relates (any, any, string) : integer

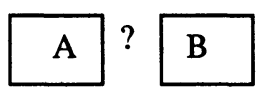

Definition 5.5: The following spatial operators also compose the GeoVisualQL Language. The resulting type is a pictorial object.

Intersection (any, any) : any

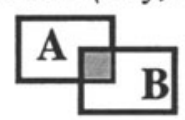

Union (any, any) : any

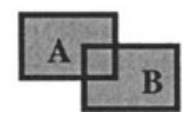

Difference (any, any) : any

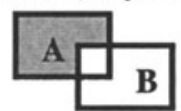

There are some others SQL functions defined in (Open GIS Consortium, SQL, 1999) but we will not represent them pictorially.

Auxiliary operators must be defined in GeoVisualQL to enhance the visual queries edition. The approach used in these editions, always consists of assigning pictorial objects to a given a spatial operator. To allow complex queries having more than one spatial operator and $\mathrm{n}$ pictorial objects to be executed we include logic operators to be used in these queries.

Operator AND: $\wedge$

Operator OR: $v$

Operator NOT: $\neg$ 
To group elements in the query construction we defined an auxiliary edition operator to be used if necessary. That is the Grouped edition operator. It is used to select which geometry entities are related with each chosen visual operator.

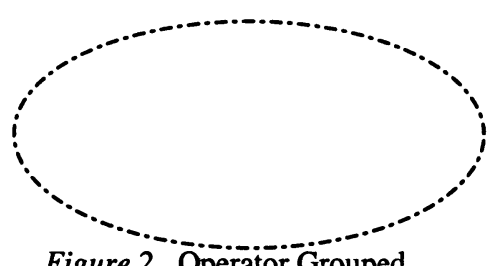

Figure 2 Operator Grouped

\subsubsection{Formal Definition of Spatial Relationships in GeoVisualQL}

The following definitions are some of the pictorial representations of the valid query clauses available in GeoVisualQL. After each of these clauses has been identified by the system as a valid clause, they will be translated to textual clauses based on the SQL specification of the Open GIS Consortium (Open GIS Consortium, SQL, 1999). It is important to note that all queries in GeoVisualQL are composed by visual elements that represent the geographic objects and by spatial visual operators that represent the spatial relationships among these entities. At this moment, we are not concerned about topologic analysis on the visual elements composing the query. We just check if there are two visual elements for each selected spatial operator. Then, the translation will be done and we will finally access the geographic database.

\section{a)Equals}

Definition: The spatial relation equals is visually represented in Figure 3, and will be valid if and only if:

EQUALS-CLAUSE $\rightarrow$ grouped (GEOMETRY1, Equals, GEOMETRY2)

Where

\section{GEOMETRY1.feature == GEOMETRY2.feature}

The where restriction is a condition to accept and translate the visual query to the SQL clause.

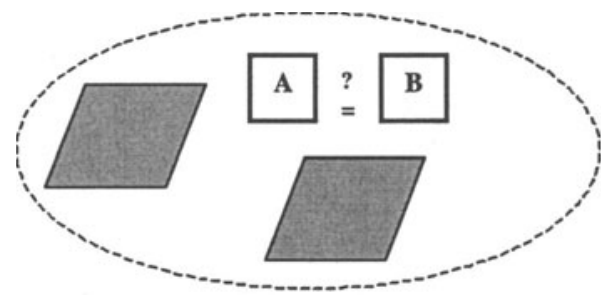

Figure 3 Representation of a visual query using the operator equals 
b) Disjoint

Definition: The disjoint operator is represented in Figure 4, and is valid if:

DISJOINT-CLAUSE $\rightarrow$ grouped (GEOMETRY1, Disjoint, GEOMETRY2)

In this case, the DISJOINT operator can be applied to two of any known geometric objects; thus, there is no need for including any restriction.

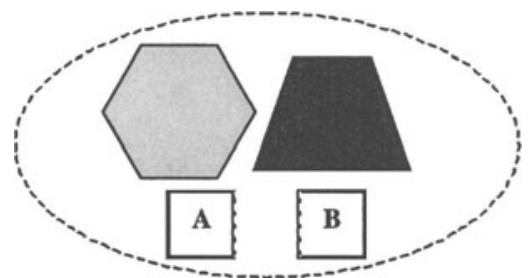

Figure 4 Symbolic representation of the visual query construction using the operator disjoint

The others operator clauses are constructed in a similar manner to these presented here and the complete definitions of these are defined in (Soares, 2002).

\subsection{GeoVisualQL Grammar Definition}

The start symbol of the language is the term STATEMENT. These are the production part of the language. These grammar definitions were defined based on (Golin, 1991).

GEOMETRY $\Rightarrow$ point $\mid$ curve| line-string| line| line-ring| polygon| multicurve| multipolygon| multiline-string| multipoint

\section{STATEMENT $\Rightarrow$ STATEMENT1 $\mid$ AND-CLAUSE $\mid$ OR-CLAUSE $\mid$ NOT- CLAUSE}

STATEMENT1 $\Rightarrow$ EQUALS-CLAUSE | DISJOINT-CLAUSE | TOUCHESCLAUSE | WITHIN-CLAUSE | OVERLAPS-CLAUSE | CROSSES-CLAUSE | INTERSECTS-CLAUSE | CONTAINS-CLAUSE | RELATE- CLAUSE | INTERSECTION-CLAUSE | DIFFERENCE-CLAUSE | UNION-CLAUSE

AND-CLAUSE $\Rightarrow$ (STATEMENT $\wedge$ STATEMENT)

OR-CLAUSE $\Rightarrow$ (STATEMENT $\vee$ STATEMENT)

NOT-CLAUSE $\Rightarrow \neg$ STATEMENT

LABEL $\Rightarrow$ string

EQUALS-CLAUSE $\rightarrow$ grouped (GEOMETRY1, Equals, GEOMETRY2)

Where

GEOMETRY1.feature $==$ GEOMETRY2.feature 
DISJOINT-CLAUSE $\rightarrow$ grouped (GEOMETRY1, Disjoint, GEOMETRY2) TOUCHES-CLAUSE $\rightarrow$ grouped (GEOMETRY1, Touches, GEOMETRY2) WITHIN-CLAUSE $\rightarrow$ grouped (GEOMETRY1, Within, GEOMETRY2) OVERLAPS-CLAUSE $\rightarrow$ grouped (GEOMETRY1, Overlaps, GEOMETRY2) CROSSES-CLAUSE $\rightarrow$ grouped (GEOMETRY1, Crosses, GEOMETRY2) Where

GEOMETRY1.type $==$ line

INTERSECTS-CLAUSE $\rightarrow$ grouped (GEOMETRY1, Intersects, GEOMETRY2) CONTAINS-CLAUSE $\rightarrow$ grouped (GEOMETRY1, Contains,GEOMETRY2) RELATE-CLAUSE $\rightarrow$ grouped (GEOMETRY1, Label, Relate, GEOMETRY2)

INTERSECTION-CLAUSE $\rightarrow$ grouped (GEOMETRY1, Intersection, GEOMETRY2)

DIFFERENCE-CLAUSE $\rightarrow$ grouped (GEOMETRY1, Difference, GEOMETRY2)

UNION-CLAUSE $\rightarrow$ grouped (GEOMETRY1, Union, GEOMETRY2)

The translation module will use these grammar definitions to produce valid textual clauses. We define in the next section an example of a query using the GeoVisualQL.

\subsection{Example of a Query in GeoVisualQL}

We shall now consider the following query where a user wants to know "Does the Capibaribe River cross the city of Recife?" The following steps will be needed to formulate the query:

1. Selection of the pictorial objects that best represent the query geographic entities.

a. After selecting a polygon element, the feature associated with it must be selected (e.g. the feature City). The city representation may either be part of the GeoVisual Standard at the interface, or it can be created and incorporated into the system for further queries. For the feature City, a specific instance must be selected and named Recife.

b. A line must be selected to represent the feature River. Following this, the instance must be identified and named Capibaribe.

2. Then the visual representation of the spatial operator Crosses must be selected and grouped together with the following two instantiated features: Recife and Capibaribe.

3. The query submission is executed. This query is illustrated in Figure 5. 


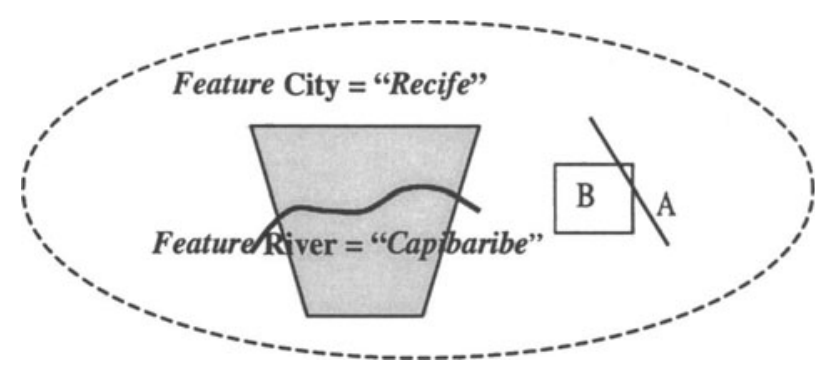

Figure 5 A Valid Query Example

After the query submission, the translation will be done by creating a textual clause in SQL, based on the specification of the OGC (Open GIS Consortium, SQL, 1999). This should be carried out by the system translation module that is now under specification.

In another example, a user could ask if the River mentioned above, Intersects any Road. This second query requires that two spatial operators are combined together by the logic operator AND, as we have specified at subsection 5.1.2. The feature Road does not need to be instantiated, and it need to be associated with the feature River named Capibaribe and with the spatial operator Intersects. So, the complete query "Does the Capibaribe River cross the City of Recife and intersect any road?" is shown in Figure 6.
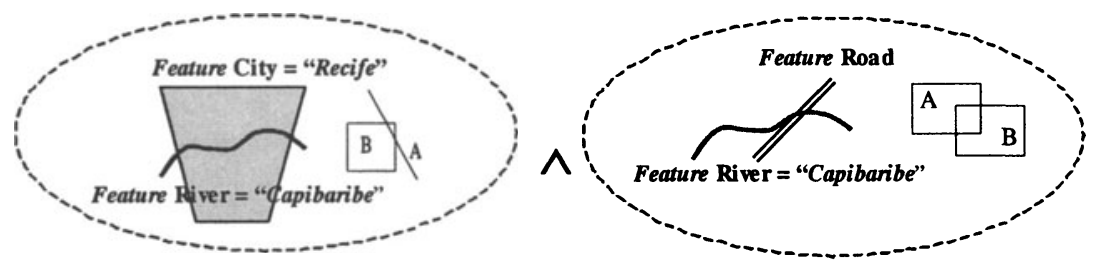

Figure 6 Another Query Example

\section{RELATED WORK}

There have been implementations of GIS research prototype that incorporate visual query languages (Blaser and Egenhofer , 2000) (Calcinelli and Mainguenaud, 1994). However, the shortcomings of these languages are two-fold. First, they do not use a standard to create the visual elements and spatial operators provided by their interface. Second, they are not based on metadata standards. Some of these proposals are briefly described bellow. 
CIGALES (Cartographical Interface Generating an Adapted Language for Extensible Systems) is a visual and declarative query language for GIS based on a Query-By-Example approach (Calcinelli and Mainguenaud, 1994). This language is visual because a query is defined by a sketch of the spatial relationships involved in the query and is declarative because it only defines the properties to be verified by the query results. It is a graphical language, which provides users with an easy and natural means of manipulating geographical data. Cigales is designed to be an upper layer of a spatial Database Management System.

VISCO (Wessel and Haarslev, 1999) is a visual spatial query system designed for extracting information from spatial information systems, specially Geographical Information Systems. VISCO supports the retrieval of interesting sets of spatial objects based on their structural, topological, metric and geometric attributes and relationships between them. The language is based on a strong naive physics metaphor for query objects and assumes a topologically-structured vector representation of the data of interest.

At a first glance, Sketch! (Meyer, 1992) may seem to be very similar to the approach taken by the CIGALES project. However, Sketch! (Meyer, 1992) aim is to explore language concepts that are best suited for non-expert users, who are not familiar with computer language concepts, but are well informed about the structure of the data they are working with. In the Sketch! user interface, context-sensitive menus guides the user through a session so that only a little knowledge of the system is required.

Spatial-Query-by-Sketch (Blaser and Egenhofer, 2000) is a sketch-based user interface to query spatial information within a GIS environment. It associates sketching with freehand drawing, rather than with the construction of geometric figures or with the composition of icons or symbols. It also allows users to sketch what they are looking for, by using a set of interpretation algorithms, needed for pen-based querying of geographical information systems. Spatial-Query-By-Sketch focuses on the graphical interaction of drawing a spatial query.

\section{FINAL CONSIDERATIONS}

In this paper we have presented an approach for visual queries in GIS, which uses a spatial metadata standard, and proposes a solution to query different spatial data in several GIS. Based on the FGDC (FGDC Standards Reference Model, 1999) description of metadata, we propose a Geographic Data Visual Standard, GeoVisual Standard and a new Geographic Visual Query Language, GeoVisualQL, to be used by GIS users. 
Users can build queries in a GIS with the information about the geographic database structures from the metadata information in interface and with the visual elements and spatial operators defined for the GeoVisualQL. The proposed framework also translates queries from the Visual Query Clause to the Textual Query Clause.

We presented in this paper GeoVisualQL grammar definitions and a query example, using some elements of the GeoVisual Standard. Currently we are specifying the Query Translation Process that will translate the visual query clause to a textual query clause based on the SQL specification of OGC (Open GIS Consortium, SQL, 1999). More information about this work can be found in (Soares, 2002).

\section{REFERENCES}

Balfanz, D. and Göbel, S. (1999). Bridging Geospatial Metadata Standards towards Distributed Metadata information Systems. The Third IEEE Meta-Data Conference. http://computer.org/conferen/proceed/meta/1999/papers/40/dbalfanz.html'

Blaser, A. D. and Egenhofer, M. J. (2000). A Visual Tool for Querying Geographic Databases. Advanced Visual Interfaces - AVI 2000, Palermo, Italy. http://www.spatial.maine.edu/ max/RC40.html

Burrough, P. and McDonnell, R. (1998). Principles of Geographical Information Systems. Oxford University Press. 1998.

Calcinelli, D. and Mainguenaud, M. (1994). Cigales: A Visual Query Language for Geographical Information System: The User Interface. International Journal of Visual Languages and Computing, Academic Press, Vol 5, pp 113-132. http://www-inf.intevry.fr/ adm_bd/Cigales/Publications/cig_publi.eng.html

Câmara, G.; Casanova M. et. al. (1996). Geographic Information Systems Anatomy. Tenth Brazilian Computer School.

Catarci, T., et.al. (1997). Visual Query Systems for Databases: Analysis and Comparison. Journal of Visual Languages and Computing, Vol. 8, N. 2, pp. 215-260. ftp://ftp.dis.uniroma1.it/pub/catarci/VQSJVLC.ps.gz

Danko, D. M. (2000). Perspectives in the Development of ISO Metadata Standards. http://www.fgdc.gov/publications/documents/metadata/nimapaper.html

Egenhofer, M. J. (1992). Why not SQL! Int. Journal Geographical Information Systems, Vol. 6, No. 2, pp 71-85.

Egenhofer, M. J. (1994). Spatial SQL: A Query and Presentation Language. IEEE Transactions on Knowledge and Data Engineering, Vol. 6, No. 1.

FGDC Standards Reference Model. (1999). Federal Geographic Data Committee. http://www.fgdc.gov/

Golin, E. J. (1991). A Method for the Specification and Parsing of Visual Languages. PhD Dissertation, Brown University, Technical Report No. CS-90-19.

Golin, E. J. and Reiss, S. P. (1990). The Specification of Visual Language Syntax. Journal of Visual Languages and Computing, Vol. 1, No.2, pp. 141-157.

Gooday, J. M. and Cohn, A. G. (1996). Visual Language Syntax and Semantics: A Spatial Logic Approach. Division of Artificial Language. University of Leeds, England. 
Guting, R. H. (1994) An Introduction to Database Systems. Special lssue on Spatial Database Systems of the VLDB Journal, Vol. 3, No. 4.

Haarslev, V. (1995). Formal Semantics of Visual Languages Using Spatial Reasoning. IEEE.

Hart, D. and Phillips, H. (1999). Metadata Primer - A "How To" Guide on Metadata Implementation. National States Geographic Information Council.

http://rat.lic.wisc.edu/metadata/metaprim.htm

Marriot, K. and Meyer, B. (1998). (Editors). Visual Language Theory. Springer Verlag.

Meyer, B. (1992). Beyond Icons. Towards New Metaphors for Visual Query Languages for Spatial Information Systems. Interfaces to Database Systems, R. Cooper (Ed.), Springer. http://www.pst.informatik.uni-muenchen.de/ bmeyer/Papers/Publications.html

Oliveira, J. L. (1997). Interface Design and Implementation for Geographic Information Systems. Phd Thesis. Unicamp, Brazil.

Open GIS Consortium Abstract Specification (1999). Version 4. http://www.opengis.org/

Open GIS Consortium, Simple Features Specification for SQL. (1999). Revision 1.1. http://www.opengis.org/

Sambura, A. (1998). Spatial Extensions to SQL. Proc. Of 16th Australian Conference on Urban and Regional Planning Information Systems (URPIS 16), pp. 359-366. Australia, Sydney.

Soares, V. G. and Salgado A. C. (2000). A Metadata-based Approach to Define a Standard to Visual Queries in GIS. . In Proceedings of the International Workshop on Interacting with Databases in conjunction with the 11th International Conference on Database and Expert Systems Applications - DEXA 2000. London, UK.

Soares, V. G. (2002) GeoVisual - A Visual Query Framework for Geographic Information Systems. PhD thesis to be concluded. Informatics Center. Federal University of Pernambuco. Brazil..

The Value of Metadata (A NSDI Report). (1999). http://www.fgdc.gov/publications/documents/metadata/metabroc.html

Tosta, N. (1998). Continuing Evolution of the National Spatial Data Infrastructure. http://www.fgdc.gov/publications/publications.html

Wang, D. and Zeevat, H. (1998). A Syntax-Directed Approach to Picture Semantics. K. Marriot and B. Meyer (Editors). Visual Language Theory. Springer Verlag.

Wessel, M. and Haarslev, V. (1999). VISCO: Bringing Visual Spatial Querying to Reality. http://kogs-www.informatik.uni-hamburg.de/ mwessel/visco-engl.html

\section{BIOGRAPHIES}

Valéria Gonçalves Soares, MSc 92, UNICAMP, is a PhD Student at Informatics Center of the Federal University of Pernambuco. She works as a researcher at the Agriculture and Livestock Research Company of Rio Grande do Norte State. Her main interests are geographic databases and visual query languages.

Ana Carolina Salgado is an Associate Professor at the Universidade Federal de Pernambuco, Brazil (Center of Informatics). She obtained her Doctorate from the University of Nice (France) in 1988. Her main research interests are in the area of non-conventional databases, specially multimedia and geographical databases, data integration on the Web and cooperative systems. Dr. Salgado has published over sixty technical articles in conference proceedings and journals. She is a member of the Brazilian Computer Society. She is the head of the Center of Informatics and also held office as head of undergraduate studies. 